\title{
Arm and Forearm Scanning Methodology for the Development of an Orthotic Device for Tetraplegic Patients
}

\author{
Branko ŠTEFANOVIČ*, Monika MICHALÍKOVÁ, Lucia BEDNARČíKOVÁ, \\ Marianna TREBUŇOVÁ, Radovan HUDÁK \\ Department of Biomedical Engineering and Measurement, Faculty of Mechanical Engineering, Tech- \\ nical University of Košice, Košice, Slovakia \\ https://doi.org/10.15221/20.06
}

\begin{abstract}
Introduction

The upper limb rehabilitation of the tetraplegic patients in the early stage of treatment consists of the grasp execution with the assistance of another person. The aim is to create an orthotic device for grip rehabilitation and to eliminate the need for assistance from rehabilitation nurse. To accomplish this target, it is necessary to obtain the positives of the patient's arm and forearm.
\end{abstract}

\section{Method}

The subjects with insufficient strength of upper limb movement are the target group of this study. Their physical state complicates the conventional way of obtaining measures and shape of the upper limb for the purpose of designing orthotic devices. Appropriate method for hand and forearm 3D scanning has been proposed to achieve a satisfactory positive model.

\section{Test/Data}

The scanning process was divided into two scans of the hand, forearm and fingers with the Artec Eva 3D scanner. It was necessary to develop specific methodology of suitable scanning positions for the purpose of this work.

\section{Results}

To hold the correct position of upper limb during scanning process is complicated, because of absence or lack of strength in the upper limb. The scanning position of the hand and forearm is the wrist extension and the thumb opposition to the fingers. This could be done with the assistance of a second person with special instruments. This scanning was performed with the focus on the dorsal part of the hand, the thumb and the radial part of the forearm. The fingers were scanned in the position with the palm placed on a cylinder surface, to achieve a scan with the fingers separated from each other. The focus during the scanning process was on the dorsal part of the fingers.

\section{Conclusions}

The required and important segments of the hand and forearm have been successfully scanned with Artec Eva 3D scanner and were used for the CAD designing of an individual rehabilitation hand orthosis. This orthotic device can later be manufactured with additive manufacturing technology.

Key words: 3D scanning, tetraplegia, positives obtaining, hand orthosis, upper limb

\section{Introduction}

Intensive rehabilitation of motor functions is important in patients in whom reduced or lost grip strength is indicated Error! Reference source not found.. These are mainly tetraplegic patients, stroke patients and patients with spinal cord injuries [3]. It is proven that the given rehabilitation helps not only to improve the gripping function of the hand, but also to the neurological renewal of the brain, which is favourable for the patient's return to active daily life Error! Reference source not found.. Rehabilitation consists in active or passive grasping and moving of standardized objects. In case of insufficient strength in the upper limb, the rehabilitation assistant assists the patient in performing specific tasks. To eliminate the need for an assistant and improve the rehabilitation itself, special mechatronic orthoses have been created to assist in the rehabilitation of motor hand functions[1],[2][3]. These devices, which have been developed in scientific institutions around the world, have been tested on patients with no or insufficient upper limb force to perform grip and have had a positive effect on overall health. At present, few mechatronic orthoses are used in practice due to their complicated construction, operation and high production costs [1][2][4][5][6]. 
For this reason, it is necessary to design and manufacture a mechatronic assistance orthosis with simple operation and individual design. To create such a tool, it is appropriate to use modern technologies ranging from 3D scanners to additive manufacturing [6],[7],[8]. Scanning the body surface using 3D scanners is, from a practical point of view, an adequate method for designing orthotic devices [8],[9],[10],[11],[12]. It is contactless and is faster, more detailed, more convenient for the patient compared to the traditional method of data collection, and in the case of portable hand-held 3D scanners, the patient's presence at a special workplace is not required [8][13]. The captured surface is highly detailed with identical anthropometric dimensions of the scanned body segment. The choice of 3D scanners for prosthetic purposes depends on the resolution, the size of the scanned area, the methodology and time of scanning, the preparation time, the price, etc. [9],[14],[15]. From the positive obtained by $3 \mathrm{D}$ scanning, a model of an orthotic device is created in the interface of suitable CAD software [12][16]. There are many purchasable or freely downloadable CAD software on the market for prosthesis and orthosis designing. Thanks to this software, a precise individual 3D design of an orthotic device is created by the method of reverse engineering, which can be subsequently produced by means of additive manufacturing [12]. Additive manufacturing technology, or 3D printing, is the production of objects by joining selected materials according to a virtual 3D model vertically divided into horizontal layers [16],[17],[18]. The biggest advantage of 3D printing is the possibility of producing structures and shapes that are too complex for production by commonly used technologies [17]. Its advantages are the reduction of the production time, a large selection of suitable materials, reduction of the amount of waste, the possibility of rapid production, etc. As a result, additive manufacturing and 3D scanning have great applications in the medical sphere [8][10].

The main goal is to design and manufacture a low-cost, individual, mechatronic orthosis produced by additive technology. This assistive orthosis will eliminate the need for a rehabilitation assistant and rehabilitate arm's gripping function by itself. The design of the orthosis will be simple and fully individual. To accomplish this target, a methodology for scanning selected segments of the arm and forearm for data obtainment is proposed.

\section{Method}

The target group of this study are subjects with insufficient strength to perform upper limb movement due to tetraplegia or other spinal cord injuries. Their physical condition complicates the obtainment of the dimensions and shape of the upper limb in a conventional manner for the purpose of orthotic device designing. It should be considered that subjects have movement restrictions not only when performing handling functions, but also when walking and moving. Taking a positive by plastering would therefore be too complicated and difficult. Another option would be to measure lengths and circumferences using hand gauges. This method would not be complicate for data acquiring, but it would be insufficient for individual orthosis designing. For these reasons, the method of obtaining the positives using the Artec Eva (Artec 3D, Luxembourg, Luxembourg) hand-held optical 3D scanner was used (Fig. 1). This makes it possible to scan the desired segment of the subject's body with high accuracy for orthotic applications.

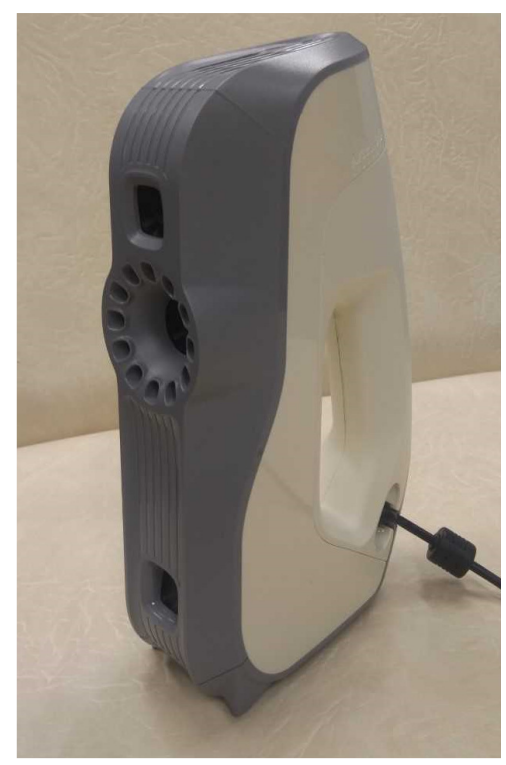

Fig. 1. Artec Eva 3D scanner. 
It is assumed that the subjects for whom the device will be intended are not able to perform movements in the wrist and hand without the help of an assistant. One of the tasks of the proposed orthosis is to provide a suitable starting position for grip performance, i.e. to fix the thumb and wrist (Fig. 2). For this reason, when scanning the hand and forearm, it is important to capture the radial part of the forearm and the dorsal area of the hand. Using this data, it will be possible to design the part of the orthosis that fixes the hand and forearm. Since flexion and extension of the fingers is necessary when gripping objects, there must be a mechanism on the orthosis that will compensate these movements instead of the residual force in the subject's fingers. This mechanism will surround the subject's fingers and to ensure an individual design, it is necessary to capture the dorsal surface of the fingers. Thanks to this model, it will be possible to design individual splints for the correct positioning of the fingers within the gripping mechanism of the orthosis.

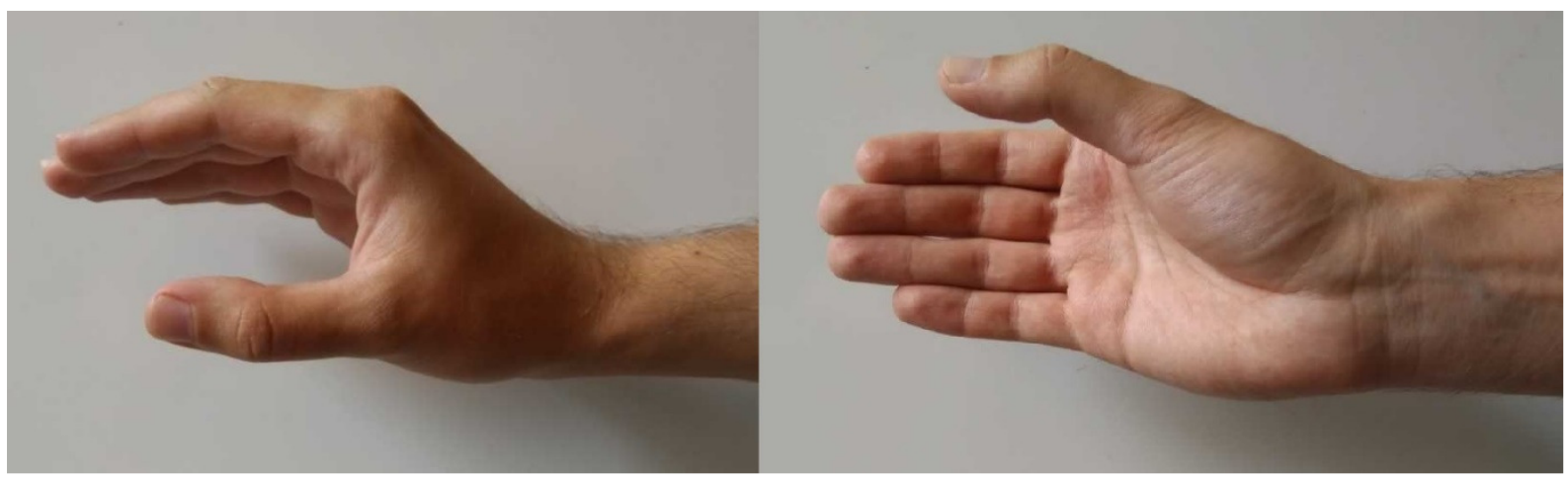

Fig. 2. Ideal scanning position of the arm and forearm.

\section{Test/Data}

To test the upper limb segment scanning method, an environment was simulated with a tetraplegic subject positioned on a hospital bed. The scanning was performed by a person trained to work with an Artec Eva hand-held optical scanner with the help of another person who assisted in the correct positioning of the subject's upper limb. The scanning process was divided into two parts, an arm and forearm scan and a finger scan.

\subsection{Arm and forearm scanning}

During this scan, it is important to capture the radial part of the forearm and the dorsal part of the hand with the thumb in opposition to the fingers and wrists at a $10-20^{\circ}$ extension. This scanning position is achieved with the help of an assistant who fixes the subject's limb in position by supporting the proximal end of the elbow bone with one hand and hook-gripping the fingers of the subject's hand with his other hand (Fig. 3). It is necessary to ensure that the wrist and thumb are in the correct position. If segment positioning is secured, another person can scan the limb.

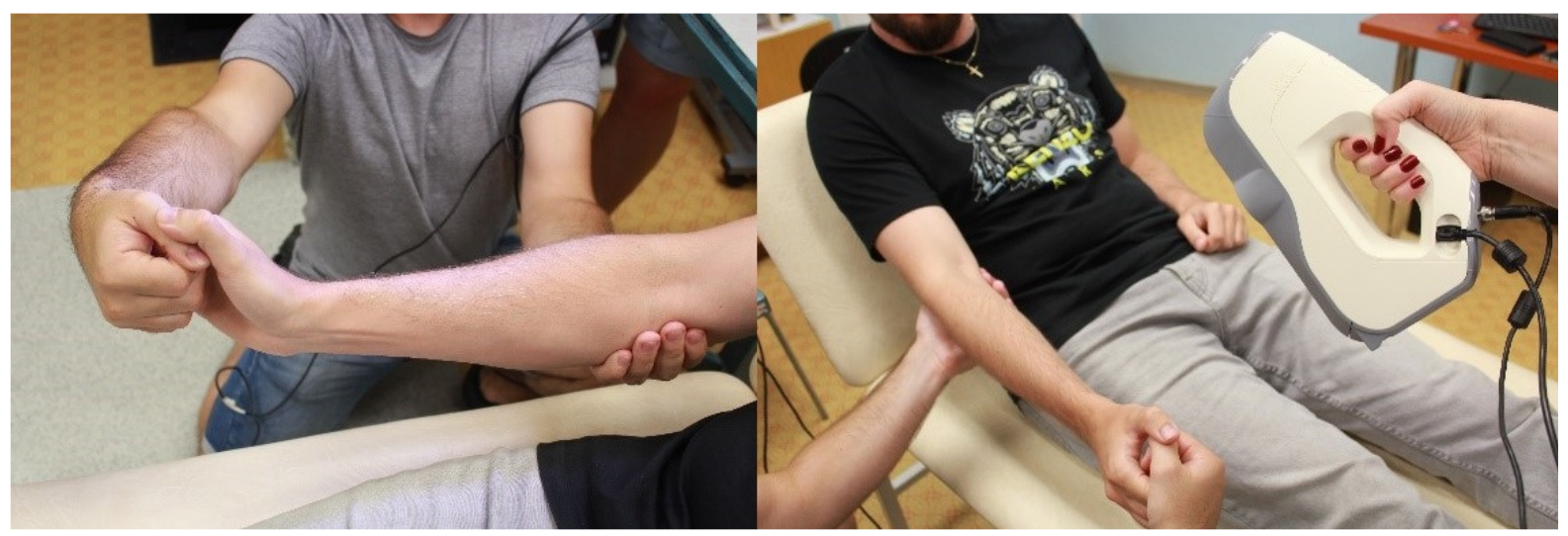

Fig. 3. Arm and forearm scanning. 


\subsection{Finger scanning}

At this stage, it is necessary to capture the dorsal surface of the fingers with MCP joints in $10-20^{\circ}$ flexion, PIP joints in approximately $10-20^{\circ}$ flexion and DIP joints in slight $1-5^{\circ}$ flexion. We secure this position when we place the palm of the subject on an oval object with a diameter of $10 \mathrm{~cm}$ and spread our fingers in mutual abduction (Fig. 4). This leaves a free space between the fingers, which is important for the finger splints designing.

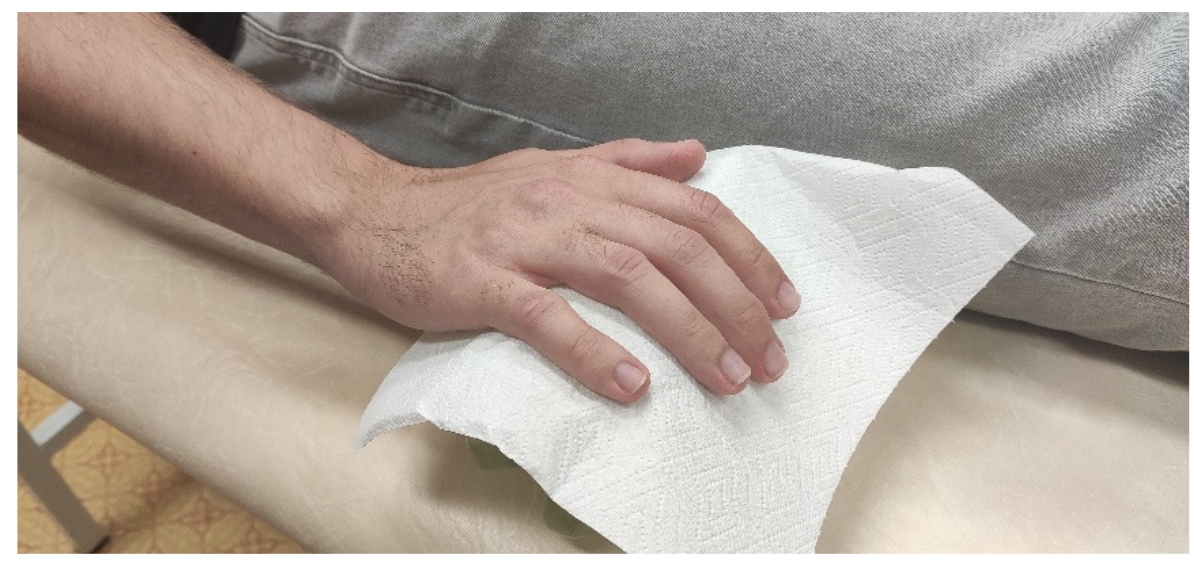

Fig. 4. Finger scanning.

\section{Results}

Hand and forearm scans were performed with different scan frequency settings: $16 \mathrm{fps}, 8 \mathrm{fps}$, 3fps and $1 \mathrm{fps}$. The first attempt after 80 seconds with a frequency of $16 \mathrm{fps}$ (frames per second) caused an error and the scanner lost tracking, so the software incorrectly connected the scanned surfaces. This could be caused by the quick manipulation with the scanner while scanning at that frequency. However, the generated model showed that important areas of the 3D model were successfully captured (Fig. 5). To eliminate or reduce the likelihood of an error, the scan frequency was reduced to 8fps and the test was repeated. In about 60 seconds, important areas of the segment were scanned without error (Fig. 5). Subsequently, the scan frequency was reduced to $3 \mathrm{fps}$ to determine the lowest frequency at which the segment could be scanned. In about 60 seconds, the area of interest was captured without side errors (Fig. 5). Finally, it was scanned at a frequency of $1 \mathrm{fps}$. At this frequency, it was too complicated to capture any part of the subject's segment. During scanning, the scanner lost tracking and the software could not process the image due to a lack of collected data. The scan results are summarized in Error! Reference source not found..

Tab. 1. Scanning results.

\begin{tabular}{|c|c|l|l|}
\hline $\begin{array}{l}\text { Frequency } \\
\text { (fps) }\end{array}$ & $\begin{array}{l}\text { Scanning time } \\
\text { length }(\mathrm{sec})\end{array}$ & Scanning errors (lost tracking) & $\begin{array}{l}\text { Applicability of the output } \\
\text { model }\end{array}$ \\
\hline 16 & 80 & Occurred & Usable \\
\hline 8 & 60 & Did not occur & Usable \\
\hline 3 & 60 & Did not occur & Usable \\
\hline 1 & $\mathrm{X}$ & Occurred & Not usable \\
\hline
\end{tabular}

From the given results it can be concluded that scanning with a frequency of $3 f p s$ is sufficient for orthosis positive obtaining. 

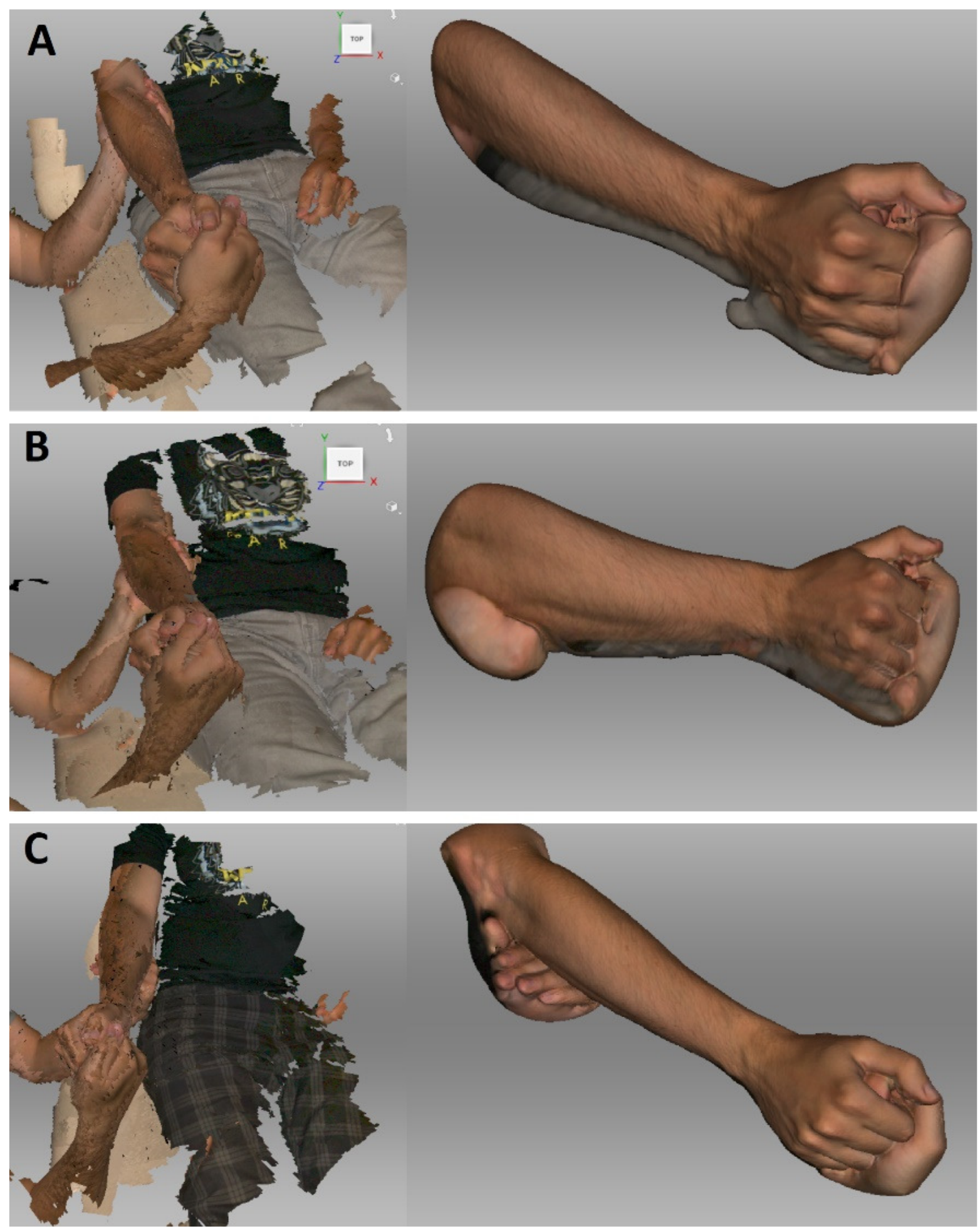

Fig. 5. 3D scans and models obtained with different scanning frequencies (A: 16fps, B: 8fps, C: 3fps).

Thanks to the information obtained from the hand and forearm scanning, the frequency was set to 3fps when scanning the fingers (Fig. 6). The scanning process took approximately 20 seconds, during which no error occurred. This confirmed that the set scanning parameters are satisfactory. 


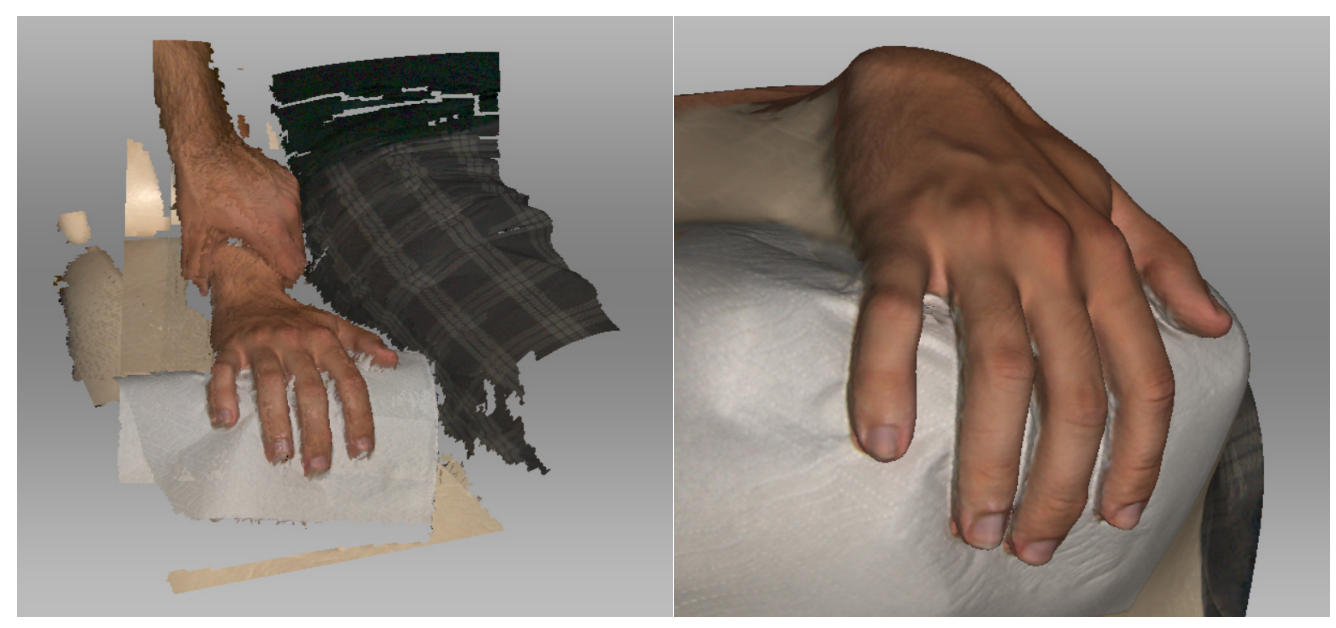

Fig. 6. 3D scan and model of the dorsal side of the arm.

Finger, hand and forearm positives generated from the 3D scanner software can be exported to a CAD software for individual splint designing (Fig. 7). Subsequently, these models can be made from a compatible plastic material using additive manufacturing technology.

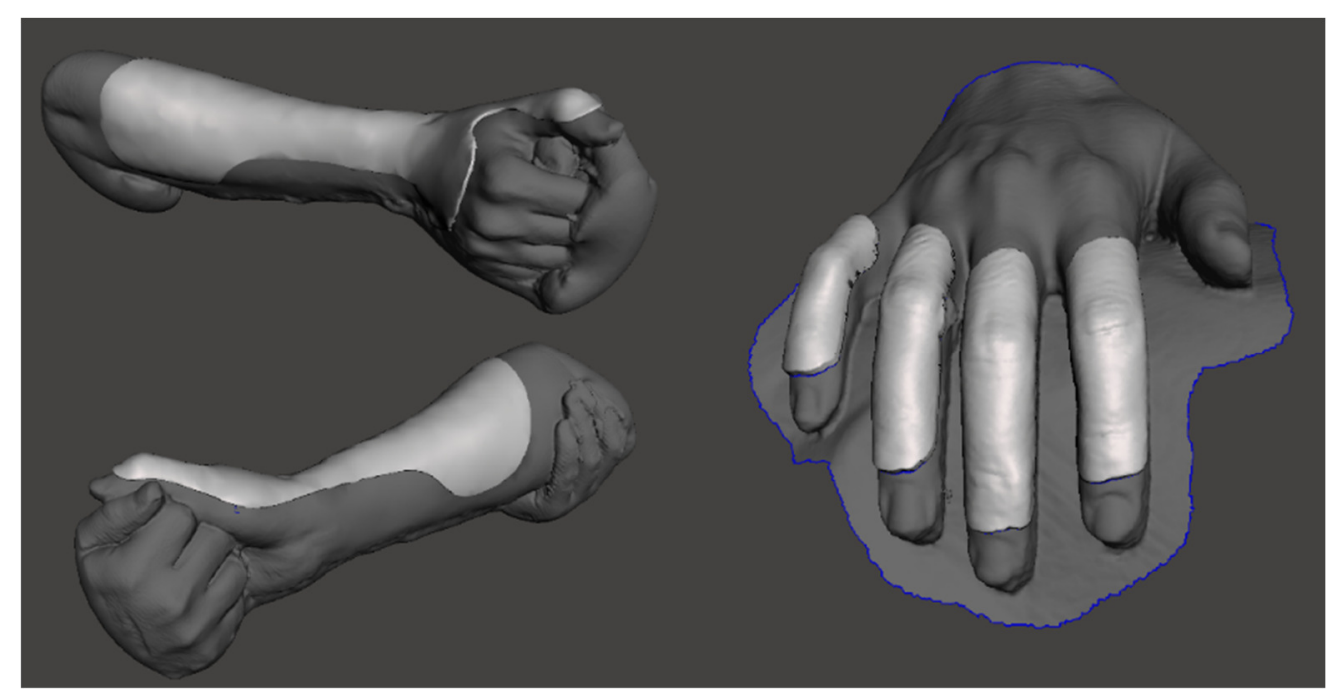

Fig. 7. Prototype splint designs for orthosis construction.

\section{Conclusions}

The proposed methodology for arm and forearm scanning proved to be very practical, fast, clean, physically undemanding and comfortable for both the subject and the scanning staff. When scanning, it is necessary to ensure that the most essential parts of the area of interest are captured with slow movement of the scanner and that the assistant firmly stabilizes the scanned segment. For this, it would be appropriate to design ergonomic fixation-stabilization aids for body segment positioning in order to eliminate the need for a scanning assistant. The test also found that a high-resolution scanner was not required for upper limb positive obtaining. It would be appropriate to compare output models of cheaper handheld 3D scanners with lower resolution. Proposed methodology can be used for obtaining models, which can be used as positives for designing 3D printable splints for mechatronic orthosis construction. In the future, given methodology will be verified by scanning 10 subjects and creating individually upper limb orthoses, which will be compared to the reference model, in order to achieve differences.

\section{Acknowledgements}

The present article was elaborated under the support received from the project APVV-19-0290, KEGA 040TUKE-4/2019, KEGA 023TUKE-4/2020, VEGA 1/0179/19 and VEGA 1/0316/18. 


\section{References}

[1] P. Heo, G. M. Gu, S. jin Lee, K. Rhee, and J. Kim, "Current hand exoskeleton technologies for rehabilitation and assistive engineering," in International Journal of Precision Engineering and Manufacturing, vol. 13, no. 5. Springer, May 04, 2012, pp. 807-824, doi: https://doi.org/10.1007/s12541$\underline{012-0107-2 .}$.

[2] I. A. Ben, Y. Bouteraa, and C. Rekik, "Design and development of 3d printed myoelectric robotic exoskeleton for hand rehabilitation," in Int. J. Smart Sens. Intell. Syst., vol. 10, no. 2, 2017, pp. 341366, doi: https://doi.org/10.21307/ijssis-2017-215.

[3] Z. Yue, X. Zhang, and J. Wang, "Hand Rehabilitation Robotics on Poststroke Motor Recovery," in Behavioural Neurology, vol. 2017, pp. 1-20, Hindawi Limited, 2017, doi: https://doi.org/10.1155/2017/3908135.

[4] T. Desplenter, Y. Zhou, B. P. Edmonds, M. Lidka, A. Goldman, and A. L. Trejos, "Rehabilitative and assistive wearable mechatronic upper-limb devices: A review," in J. Rehabil. Assist. Technol. Eng., vol. 7, Jan. 2020, p. 205566832091787, doi: https://doi.org/10.1177/2055668320917870.

[5] A. A. Portnova, G. Mukherjee, K. M. Peters, A. Yamane, and K. M. Steele, "Design of a 3D-printed, open-source wrist-driven orthosis for individuals with spinal cord injury," in PLoS One, vol. 13, no. 2, Feb. 2018, doi: https://doi.org/10.1371/journal.pone.0193106.

[6] H. J. Yoo, S. Lee, J. Kim, C. Park, and B. Lee, "Development of 3D-printed myoelectric hand orthosis for patients with spinal cord injury," J. Neuroeng. Rehabil., vol. 16, no. 1, Dec. 2019, pp. 1-14, doi: https://doi.org/10.1186/s12984-019-0633-6.

[7] H. Lin, L. Shi, and D. Wang, "A rapid and intelligent designing technique for patient-specific and 3D-printed orthopedic cast," in 3D Print. Med., vol. 2, no. 1, Dec. 2016, pp. 1-10, doi: https://doi.org/10.1186/s41205-016-0007-7.

[8] R. K. Chen, Y. an Jin, J. Wensman, and A. Shih, "Additive manufacturing of custom orthoses and prostheses-A review," in Additive Manufacturing, vol. 12. Elsevier B.V., Oct. 01, 2016, pp. 77-89, doi: https://doi.org/10.1016/j.addma.2016.04.002.

[9] D. Palousek, J. Rosicky, D. Koutny, P. Stoklásek, and T. Navrat, "Pilot study of the wrist orthosis design process," in Rapid Prototyp. J., vol. 20, no. 1, 2014, pp. 27-32, doi: https://doi.org/10.1108/RPJ-03-2012-0027.

[10]P. Gil, C. Mateo, and F. Torres, "3D visual sensing of the human hand for the remote operation of a robotic hand," in Int. J. Adv. Robot. Syst., vol. 11, no. 1, Feb. 2014, pp. 1-13, doi: https://doi.org/10.5772\%2F57525.

[11] G. Baronio, S. Harran, and A. Signoroni, "A Critical Analysis of a Hand Orthosis Reverse Engineering and 3D Printing Process," in Appl. Bionics Biomech., vol. 2016, 2016, pp. 1-7, doi: https://doi.org/10.1155/2016/8347478.

[12]F. Buonamici et al., "A CAD-based procedure for designing 3D printable arm-wrist-hand cast," in Comput. Aided. Des. Appl., vol. 16, no. 1, 2018, pp. 25-34, doi: 10.14733/cadaps.2019.25-34

[13]D. Palousek, J. Rosicky, D. Koutny, P. Stoklásek, and T. Navrat, "Pilot study of the wrist orthosis design process," in Rapid Prototyp. J., vol. 20, no. 1, 2014, pp. 27-32, doi: https://doi.org/10.1108/RPJ-03-2012-0027.

[14]P. Volonghi, a. Signoroni, and g. Baronio, "3D Scanning for Hand Orthotic Applications: A Comparative Assessment between Static and Real-Time Solutions," Nov. 2016, pp. 61-69, doi: https://doi.org/10.15221/16.061.

[15] "(PDF) 3D digitalization of the human body for use in orthotics and prosthetics." https://www.researchgate.net/publication/281510829 3D digitalization of the human body for use in orthotics and prosthetics (accessed Aug. 13, 2020).

[16]A. Willis, J. Speicher, and D. B. Cooper, "Rapid prototyping 3D objects from scanned measurement data," in Image Vis. Comput., vol. 25, no. 7, Jul. 2007, pp. 1174-1184, doi: https://doi.org/10.1016/j.imavis.2006.06.011.

[17]H. Zhou and S. B. Bhaduri, "3D printing in the research and development of medical devices," in Biomaterials in Translational Medicine: A Biomaterials Approach, Elsevier, 2018, pp. 269-289, doi: https://doi.org/10.1016/B978-0-12-813477-1.00012-8.

[18] M. Javaid and A. Haleem, "Additive manufacturing applications in medical cases: A literature based review," in Alexandria J. Med., vol. 54, no. 4, Dec. 2018, pp. 411-422, doi: https://doi.org/10.1016/i.ajme.2017.09.003. 